\title{
HERITABILITY OF HIERARCHICAL STRUCTURAL BRAIN NETWORK
}

\author{
Moo K. Chung*, Zhan Luo, Nagesh Adluru, Andrew L. Alexander, Davidson J. Richard, H. Hill Goldsmith \\ University of Wisconsin-Madison, USA
}

\begin{abstract}
We present a new structural brain network parcellation scheme that can subdivide existing parcellations into smaller subregions in a hierarchically nested fashion. The hierarchical parcellation was used to build multilayer convolutional structural brain networks that preserve topology across different network scales. As an application, we applied the method to diffusion weighted imaging study of 111 twin pairs. The genetic contribution of the whole brain structural connectivity was determined. We showed that the overall heritability is consistent across different network scales.
\end{abstract}

\section{INTRODUCTION}

In the usual brain connectivity studies, the whole brain is often parcellated into $p$ disjoint regions, where $p$ is usually 116 or less [1, 2]. For instance, Anatomical Automatic Labeling (AAL) parcellation provides 116 labels for all the cortical and subcortical structures (Figure 1) [1]. Subsequently, either functional or structural information is overlaid on top of AAL and $p \times p$ connectivity matrices that measure the strength of connectivity between brain regions are obtained. The major shortcoming of using the existing parcellations including AAL is the lack of refined spatial resolution. Even if we detected connectivity differences between large chunk of brain regions, it is not possible to localize what parts of parcellations are affected without additional analysis. There is a strong need to develop a higher resolution parcellation scheme.

Brain networks are fundamentally multiscale. Intuitive and palatable biological hypothesis is that brain networks are organized into hierarchies [3]. A brain network at any particular sale might be subdivided into subnetworks, which can be further subdivided into smaller subnetworks in an iterative fashion. Unfortunately, many parcellation schemes give raise to conflicting topological structures of the parcellation from one scale to the next. The topological structure of parcellation at one particular scale may not carry over to different scales $[2,3]$. There is a need to develop a hierarchical parcellation scheme that provide a consistent network analysis results and interpretation regardless of the choice of scale.

Correspondence should be sent to M.K.C (mkchung@wisc.edu). This work was supported by NIH research grants R01 EB022856, R01 MH101504, P30 HD003352, U54 HD09025.
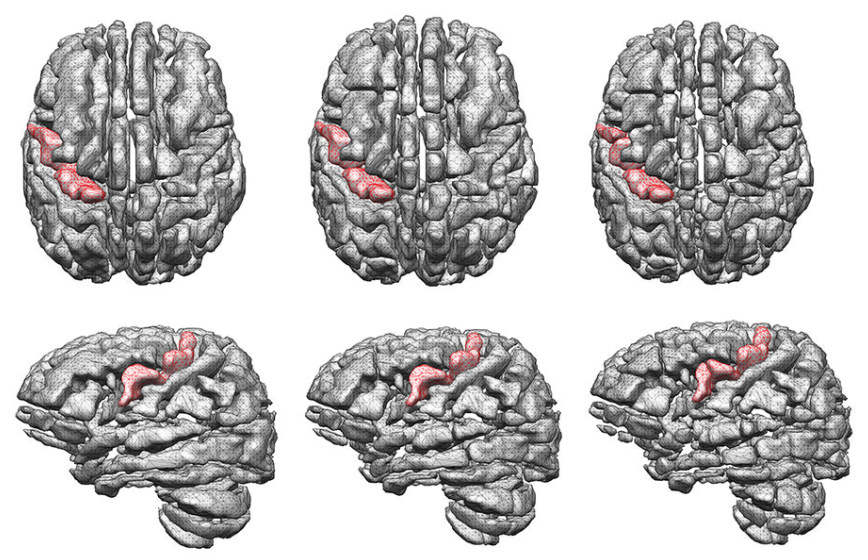

Fig. 1. Left: AAL parcellation with 116 regions. Red region is the left precentral gyrus. Middle: the second layer of the hierarchical parcellation with $2 \times 116$ regions. Each AAL parcellation is subdivided into two disjoint regions. Right: the third layer of the hierarchical parcellation with $4 \times 116$ regions. Artificial gaps between subparcellations are introduced for visualization purpose only.

In this study, we propose a new hierarchical parcellation scheme based on the Courant nodal domain theorem [4]. The proposed method is related to graph cuts [5] and spectral clustering $[6,7]$ based parcellation schemes previous used in parcellating the resting-state functional magnetic resonance imaging (fMRI). However, in all these studies, parcellations are not hierarchical or nested so they produce conflicting topology over different network scales. Unlike previous approaches, our approach provides hierarchical nestedness and, thus, preserves topology across different spatial resolutions.

As an application, the proposed method was applied to diffusion weighted imaging (DWI) study of 111 twin pairs in determining the statistical significance of the genetic contribution of the whole brain structural connectivity.

\section{HIERARCHICAL CONNECTIVITY}

Courant nodal domain theorem. For Laplacian $\Delta$ in a compact domain $\mathcal{M} \subset \mathbb{R}^{3}$, consider eigenvalues

$$
0=\lambda_{0}<\lambda_{1} \leq \lambda_{2} \leq \cdots
$$




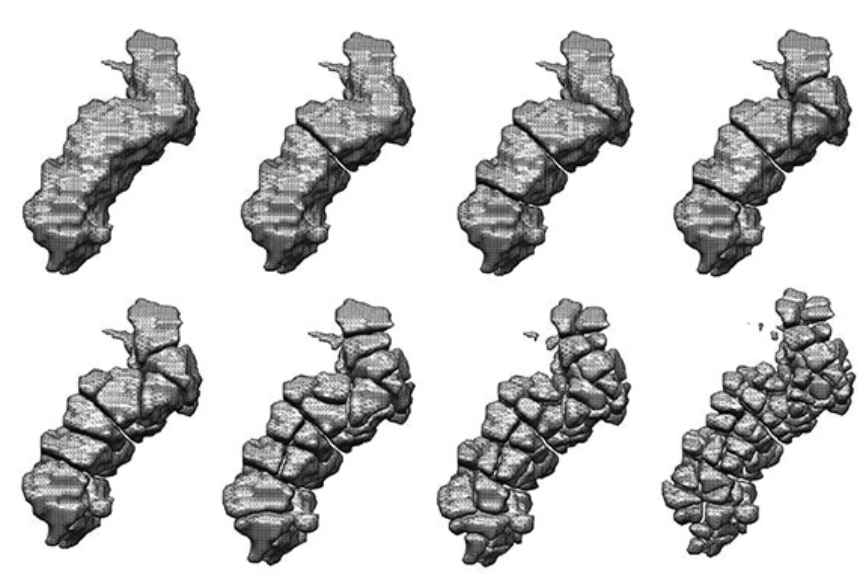

Fig. 2. Hierarchical parcellation of the left precentral gyrus shown in Figure 1) up to the 8-th layer. At the 8-th layer, we have $2^{8-1}=128$ parcellations of the gyrus. The hierarchical parcellation continues till every voxel is a parcellation.

and eigenfunctions $\psi_{0}, \psi_{1}, \psi_{2}, \cdots$ satisfying

$$
\Delta \psi_{j}(p)=\lambda_{j} \psi_{j}(p)
$$

We then have $\psi_{0}(p)=1 / \sqrt{\mu(\mathcal{M})}$, where $\mu(\mathcal{M})$ is the volume of $\mathcal{M}$. From the orthogonality of eigenfunctions, we have

$$
\int_{\mathcal{M}} \psi_{0}(p) \psi_{1}(p) d \mu(p)=0,
$$

Thus, $\psi_{1}$ must be take positive and negative values. The Courant nodal domain theorem [4] further states that $\psi_{1}$ divides $\mathcal{M}$ into two disjoint regions by the nodal surface boundary $\psi_{1}(p)=0$. When the domain is discretized as a 3D graph, the second eigenfunction $\psi_{1}$ is called the Fiedler vector. It is often used in spectral clustering and graph cuts [5, 8]. Applying iteratively the nodal domain theorem, we can hierarchically partition $\mathcal{M}$ in a nested fashion.

Hierarchical parcellation. The Courant nodal domain theorem is discretely applied to the AAL parcellation as follows. We first convert the binary volume of each parcellation in AAL into a 3D graph by taking each voxel as a node and connecting neighboring voxels. Using the 18-connected neighbor scheme, we connect two voxels only if they touch each other on their faces or edges. If voxels are only touching at their corner vertices, they are not considered as connected. This results in an adjacency matrix and the 3D graph Laplacian. The computed Fiedler vector is then used to partition each AAL parcellation into two disjoint regions (Figures 1 and 2). For each disjoint subregion, we further recompute the Fiedler vector restricted to the subregion. This binary partition process iteratively continues till all the partitions are voxels. We are doubling the number of parcellations at each iteration. There are a total of $p=116$ parcellations in layer 1 and $2 \cdot 115$
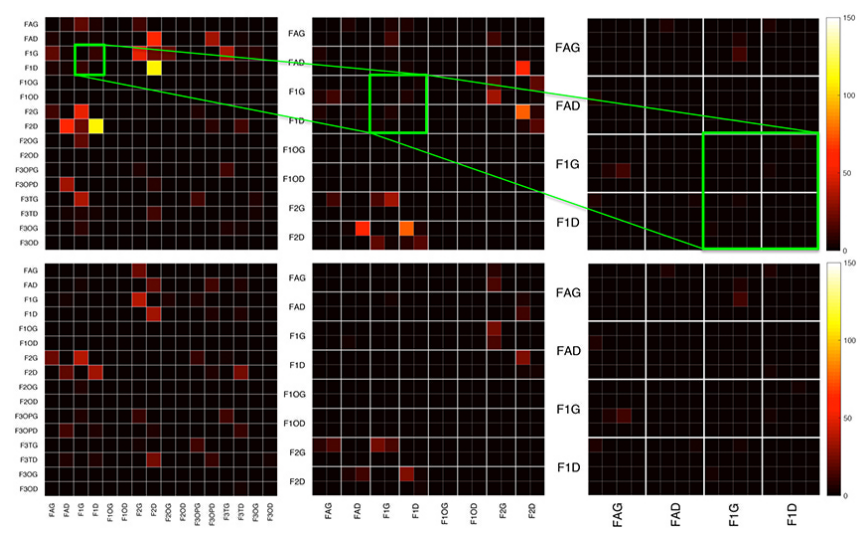

Fig. 3. The hierarchical connectivity matrices of MZ- (top) and DZ-twins (bottom). The parts of connectivity matrices of the layers 1, 2 and 3 are shown. They form a layered convolutional network, where the convolution is defined as the sum of tracts between sub-parcellations.

parcellations in layer 2 . At the $i$-th layer, there are $2^{i-1} \cdot 116$ parcellations. In our study, we were able to construct 20-layer nested hierarchical parcellations all the way to the voxel-level.

Convolutional network. At the each layer of the hierarchical parcellation, we counted the total number of white matter fiber tracts connecting parcellations as a measure of connectivity. The resulting connectivity matrices form a convolutional network. Let $S_{j k}^{i}$ denote the total number of tracts between parcellations $\mathbf{R}_{j}^{i}$ and $\mathbf{R}_{k}^{i}$ at the $i$-th layer. The connectivity $S_{j k}^{i}$ at the $i$-th layer is then the sum of connectivities at the $(i+1)$-th layer (Figure 3), i.e.,

$$
S_{j k}^{i}=\sum_{\mathbf{R}_{l}^{i+1} \subset \mathbf{R}_{j}^{i} \mathbf{R}_{m}^{i+1} \subset \mathbf{R}_{k}^{i}} S_{l m}^{i+1} .
$$

The sum is taken over every subparcellation of $\mathbf{R}_{j}^{i}$ and $\mathbf{R}_{k}^{i}$.

\section{APPLICATION}

Subjects. Participants were part of the Wisconsin Twin Project [9]. 58 monozygotic (MZ) and 53 same-sex dizygotic (DZ) were used in the analysis. Twins were scanned in a 3.0 Tesla GE Discovery MR750 scanner with a 32-channel receive-only head coil. Diffusion tensor imaging was performed using a three-shell diffusion-weighted, spin-echo, echo-planar imaging sequence. A total of 6 non-DWI $(b=0 \mathrm{~s} \cdot \mathrm{mm} 2)$ and 63 DWI with non-collinear diffusion encoding directions were collected at $\mathrm{b}=500,800,2000$ (9, 18, 36 directions). Other parameters were TR/TE $=8575 / 76.6 \mathrm{~ms}$; parallel imaging; flip angle $=90^{\circ}$; isotropic $2 \mathrm{~mm}$ resolution $(128 \times 128$ matrix with $256 \mathrm{~mm}$ field-of-view).

Image preprocessing follows the pipeline established in [10]. FSL were used to correct for eddy current related dis- 
MZ
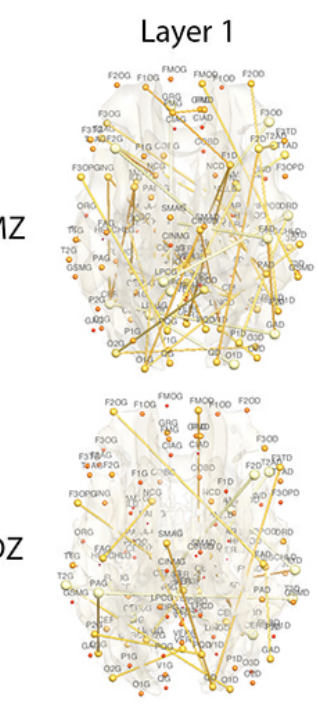

$\mathrm{HI}$

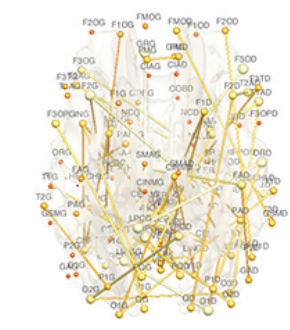

Layer 2
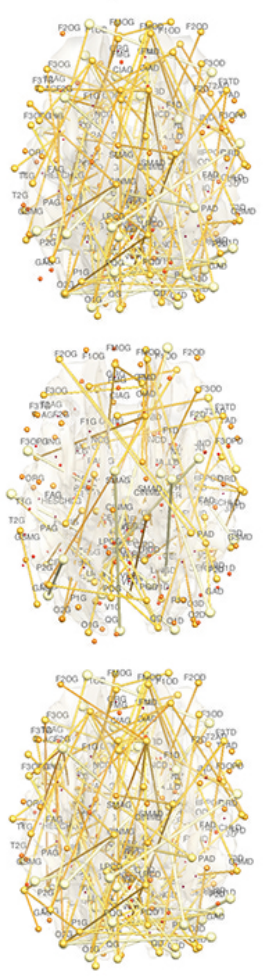

Layer 3
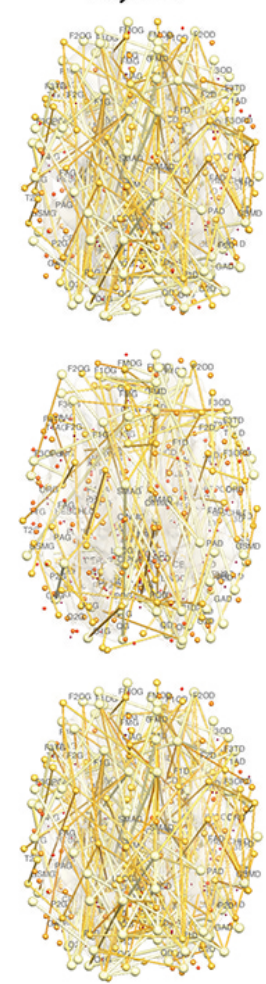

Layer 4
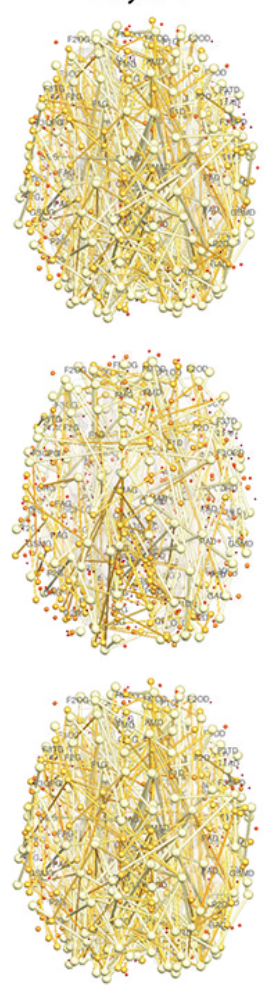

Layer 5
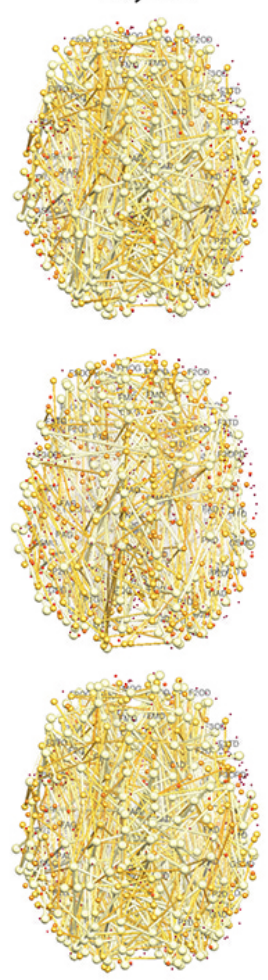

Layer 6
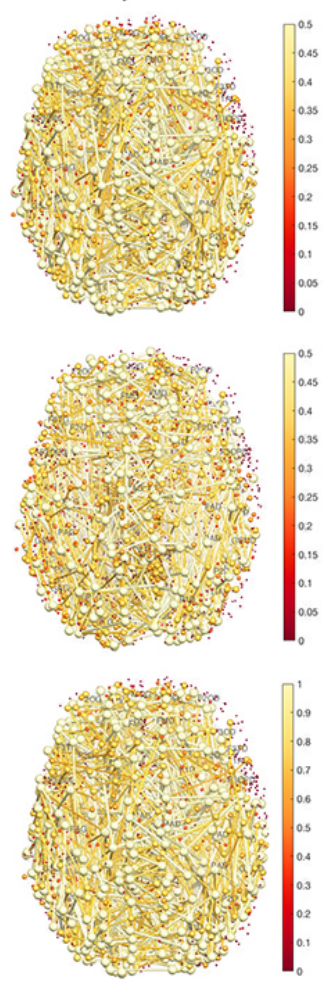

Fig. 4. Top, middle: Edge colors are Spearman's rank correlations thresholded at 0.3 for MZ- and DZ-twins for different layers. Node colors are the maximum correlation of all the connecting edges. Bottom: Edge colors are the heritability index (HI). Node colors are the maximum HI of all the connecting edges. MZ-twins show higher correlations compared to DZ-twins. The node and edge sizes are proportionally scaled.

tortions, head motion and field inhomogeneity. Estimation of the diffusion tensors at each voxel was performed using nonlinear tensor estimation in CAMINO. DTI-TK was used for constructing the study-specific template. Spatial normalization was performed tensor-based white matter alignment using a non-parametric diffeomorphic registration method. Each subject's tractography was constructed using TEND algorithm, and tracts were terminated at FA-value less than 0.2 and deflection angle greater than 60 degree.

Heritability Index. We are interested in knowing the extent of the genetic influence on the structural brain network of these participants and determining its statistical significance over different parcellation scales. For quantification, we used the heritability index (HI), which determines the amount of variation due to genetic influence in a population. HI is often estimated using Falconer's formula as a baseline [11]:

$$
\mathrm{HI}=2\left(\rho_{\mathrm{MZ}}-\rho_{\mathrm{DZ}}\right),
$$

where $\rho_{\mathrm{MZ}}$ and $\rho_{\mathrm{DZ}}$ are the pairwise correlation between MZand and same-sex DZ-twins (Figure 4). For discrete tract counts, it is more reasonable to use Spearman's correlation than Pearson's correlation. The Pearson's correlation does not work well with discrete tract count measures that often do not necessarily scale at the constant rate across different subjects and parcellations. Note Spearman's correlation is Pearson's correlation between the ordered tract counts.

Exact Topological Inference. We determined the statistical significance of $\mathrm{HI}$ using the exact topological inference [11]. Consider weighted networks $G^{1}$ and $G^{2}$. Let $G_{\lambda}^{1}$ and $G_{\lambda}^{2}$ be the binary networks obtained by thresholding $G^{1}$ and $G^{2}$ at correlation $\lambda$. Let $B$ be a monotonic graph function such that

$$
B\left(G_{\lambda_{1}}^{1}\right) \leq B\left(G_{\lambda_{1}}^{2}\right) \text { and } B\left(G_{\lambda_{2}}^{1}\right) \leq B\left(G_{\lambda_{2}}^{2}\right)
$$

for $\lambda_{1} \leq \lambda_{2}$. The number of connected components (Betti-0 number) often used in persistent homology is such a function. The test statistic

$$
D_{q}=\sup _{1 \leq j \leq q}\left|B\left(G_{\lambda_{j}}^{1}\right)-B\left(G_{\lambda_{j}}^{2}\right)\right|
$$

is used to determine the statistical significance. The thresholds $\lambda_{j}$ are chosen uniformly in $[0,1]$ at 0.01 increment. The $p$-value under the null hypothesis of no network difference is 


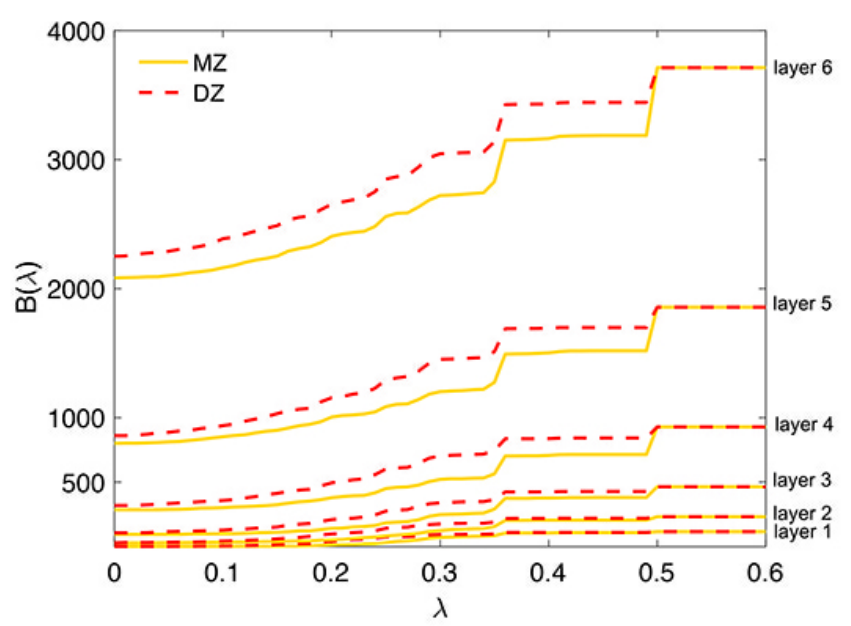

Fig. 5. Betti-0 plots. The number of connected components (vertical) over the thresholded correlation values (horizontal) at each layer. The plots scale up over different layers resonably well. The sudden changes in the topological structure of network match up at the same correlation values.

then computed using [11]

$$
P\left(D_{q} / \sqrt{2 q} \geq d\right) \approx 2 \sum_{i=1}^{\infty}(-1)^{i-1} e^{-2 i^{2} d^{2}} .
$$

Results. We are only showing results up to the 6-th layer, which has 3712 parcellations (61 voxels per parcellation in average). Beyond 6 layers, the individual parcellation was too small to be easily interpretable or visualize (Figure 4). At each layer, we performed the exact topological inference and obtained very consistent results. The $p$-values are less than $10^{-12}$ for each layer indicating the strong overall genetic contribution on the structural brain networks. Figure 5 shows the Betti-0 plots [11], which show the change of the number of connected components $B\left(G_{\lambda}\right)$ over correlation threshold values $\lambda$ for MZ- (solid yellow) and DZ-twins (dotted red). The sudden topological changes are occurring at the almost same correlation values regardless of the scale of the network.

\section{CONCLUSION}

We have developed new nested hierarchical structural brain parcellation and network methods. The methods were used in determining the genetic contribution of anatomical connectivity. The framework provides the topologically consistent statistical inference results regardless of the scale of the parcellation used. It is hoped the proposed parcellation and network construction frameworks will provide more consistent and robust network analysis across different studies and populations without concern for spatial resolution.

\section{REFERENCES}

[1] N. Tzourio-Mazoyer, B. Landeau, D. Papathanassiou, F. Crivello, O. Etard, N. Delcroix, B. Mazoyer, and M. Joliot, "Automated anatomical labeling of activations in spm using a macroscopic anatomical parcellation of the MNI MRI single-subject brain," NeuroImage, vol. 15, pp. 273-289, 2002.

[2] A. Zalesky, A. Fornito, I.H. Harding, L. Cocchi, M. Yücel, C. Pantelis, and E.T. Bullmore, "Wholebrain anatomical networks: Does the choice of nodes matter?," NeuroImage, vol. 50, pp. 970-983, 2010.

[3] R.F. Betzel and D.S. Bassett, "Multi-scale brain networks," Neuroimage, vol. in press, 2017.

[4] R. Courant and D. Hilbert, Methods of Mathematical Physics, Interscience, New York, English edition, 1953.

[5] X. Shen, X. Papademetris, and R.T. Constable, "Graphtheory based parcellation of functional subunits in the brain from resting-state fMRI data," Neuroimage, vol. 50, pp. 1027-1035, 2010.

[6] R.C. Craddock, G.A. James, P.E. Holtzheimer, X.P. Hu, and H.S. Mayberg, "A whole brain fMRI atlas generated via spatially constrained spectral clustering," Human Brain Mapping, vol. 33, pp. 1914-1928, 2012.

[7] A. Pepe, G. Auzias, F. De Guio, F. Rousseau, D. Germanaud, J.-F. Mangin, N. Girard, O. Coulon, and J. Lefèvre, "Spectral clustering based parcellation of fetal brain MRI," in IEEE International Symposium on Biomedical Imaging (ISBI), 2015, pp. 152-155.

[8] M.K. Chung, S. Seo, N. Adluru, and H.K. Vorperian, "Hot spots conjecture and its application to modeling tubular structures," in International Workshop on Machine Learning in Medical Imaging, 2011, vol. 7009, pp. 225-232.

[9] H.H. Goldsmith, K. Lemery-Chalfant, N.L. Schmidt, C.L. Arneson, and C.K. Schmidt, "Longitudinal analyses of affect, temperament, and childhood psychopathology," Twin Research and Human Genetics, vol. 10, pp. 118-126, 2007.

[10] M.K. Chung, J.L. Hanson, L. Adluru, A.L. Alexander, R.J. Davidson, and S.D. Pollak, "Integrative structural brain network analysis in diffusion tensor imaging," Brain Connectivity, vol. 7, pp. 331-346, 2017.

[11] M.K. Chung, V. Vilalta-Gil, H. Lee, P.J. Rathouz, B.B. Lahey, and D.H. Zald, "Exact topological inference for paired brain networks via persistent homology," in Information Processing in Medical Imaging (IPMI), Lecture Notes in Computer Science, 2017, vol. 10265, pp. 299-310. 